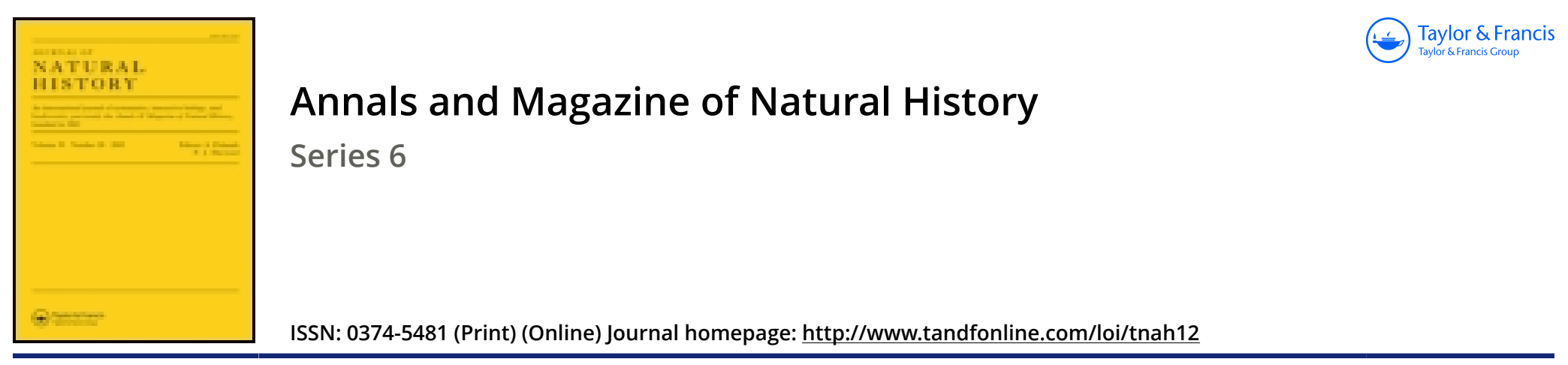

\title{
XXI.-A revision of the British Jurassic Bryozoa.-Part IV. The genera Reptomultisparsa and Diastopora
}

\author{
J.W. Gregory D.Sc. F.G.S.
}

To cite this article: J.W. Gregory D.Sc. F.G.S. (1896) XXI._A revision of the British Jurassic Bryozoa.-Part IV. The genera Reptomultisparsa and Diastopora, Annals and Magazine of Natural History, 17:98, 151-155, DOI: 10.1080/00222939608680340

To link to this article: http://dx.doi.org/10.1080/00222939608680340

曲 Published online: 06 Oct 2009.

Submit your article to this journal

Џll Article views: 3

Q View related articles $₫$

Citing articles: 1 View citing articles 
XXI.-A Revision of the British Jurassic Bryozoa.-Part IV. The Genera Reptomultisparsa and Diastopora. By J. W. Gregory, D.Sc., F.G.S.

[Continued from $p, 48$.

Family Tubuliporidæ (concluded).

Genus Reptomultisparsa, d'Orbigny, 1852.

Diagnosis.-Tubuliporidæ in which the zoarium is encrusting and consists of thick multilamellar sheets. The zocecia are cylindrical and parallel to the surface upon which the zoarium has grown. The peristome is flush or slightly raised.

Type species : $R$. microstoma (Mich.), syn. R. diluviana, Edw. \& Mich. (non Lamx.).

Affinities.-This genus was founded by d'Orbigny for thick multilamellar species allied to Berenicea. The division seems to me convenient. The first of the five species referred to the genus by d'Orbigny, which is accordingly here taken as the type, is the Diastopora diluviana, Edw. \& Mich. (non Lamx.). This, however, I regard as the same as Michelin's Diastopora microstoma.

\section{Reptomultisparsa undulata (Michelin), 1846.}

Diastopora undulata, Michelin, 1846, Icon. Zooph. p. 242, pl. Ivi. fig. 15.

Berenicea undulata, d'Orbigny, 1852, Pal. franç., Terr. crét. t. v. p. 860.

Berenicea microstoma (non Mich.), Haime, 1854, Bry. Jur., Mém. Soc. géol. France, sér. 2, t. v. p. 178, pl. vii. fig. 3.

Diastopora microstoma, var. connectens, Vine, 1884, Polyz. Rich. Bor., Quart. Journ. Geol. Soc. vol. xl. p. 789.

Diagnosis.-Zoarium in large, thick, irregular encrusting sheets, which in the adult completely bury the shell they encrust. Young forms are flabelliform.

Zocecia long or medium in length. Young zocecia are seen throughout. The more adult peripheral zocecia are crowded, and thus not wholly seen. Surface traversed by sinuous raised ridges.

Peristomes slightly raised, irregularly distributed.

Formula.-1/1" $02 i 0$.

Distribution.-England : Great Oolite, Richmond boring, Bradford Clay, Bradtord. Foreign: Bajocian and Bathonian, France and Germany. 


\section{Genus Diastopora, Lamouronx, 1821 (emended).}

Diagnosis.-Tubuliporidæ in which the zoarium is erect and foliaceous. (The zoarium consists of simple fronds or may be split up into multifid segments, or may grow in hemispherical masses by the crumpling of the fronds, or may be cylindrical, or may be reteporiform.) The zoarium is unilaminate or bilaminate. The zoceia are tubular. The peristomes are flush or raised only a small proportion of the length of the zoceia. The oøcia are closed, slightly enlarged zoceia.

Type species : D. foliacea, Lamouroux.

\section{Diastopora foliacea, Lamouroux, 1821 .}

Diastopora foliacea (pars), Lamouroux, 1821, Expos. méth. p. 42, pl. Ixxiii. figs. 1,2 (non figs. 3,4 ).

Eschara foliacea, Bronn, 1835, Leth. Geogn. Bd. i. p. 241.

Elea foliacea, Brauns, 1879, Bry. mitt. Jura, Metz, Zeit. deut. geol. Ges. Bd. xxxi. p. 313, pl. vi.

Berenicea foliacea, Vine, 1880, Review Diastoparidæ, Quart. Journ. Geol. Soc. vol. xxxvi. p. 357.

Diastopora Eudesiana, M.-Edwards, 1838, op. cit. p. 225, pl. xiv. fig. 1.

Bidiastopora Eudesia, d'Orbigny, 1849, Prod. Pal. t. i. p. 317.

Mesenteripora Eudesia, d’Orbigny, 1852, Pal. franç., Terr. crét. t. v. p. 808.

Berenicea Eudesiana, Vine, 1880, Review Diastoporidæ, Quart. Journ. Geol. Soc. vol. xxxvi. p. 357.

Bidiastopora maandrina, d'Orbigny, 1849, Prod. Pal. t. i. p. 289.

Diastopora mettensis, Haine, 1854, Bry. Jur., Mém. Soc. géol. France, sór. 2, t. v. p. 190 , pl. viii. fig. 10.

Bidiastopora macropora, d'Orbigny, 1852, Pal. franç., Terr. crét. t. v. p. 799 .

Diastopora macropora, Haime, 1854, op. cit. p. 191.

Diastopora petaloides, Waagen, Zone Amm. Sowerbyi, Geogn. Pal. Beitr. Bd. i. Heft 3, p. 645.

Diagnosis.-Zoarium loose and open, the fronds being generally broad, thin, and only slightly contorted; bilaminate.

Zocecia visible throughont, the zoceia being long and the apertures distant from one another and irregular in arrangement; the zoocia are regularly cylindrical.

Peristomes well raised, giving a rough aspect to the zoarium.

Gonocia simple closed zocecia.

Formula. -203 fr $i 0$.

Distribution. - British : Inferior Oolite - Cornbrash. Foreign: Bajocian, Germany; Bathonian, France. 


\section{Diastopora Davidsoni, Haime, 1854.}

Diastopora Davedsoni, Haime, 1851, Bry. Jur., Mem. Soc. geol. France, sér. 2, t. v. p. 185 , pl. viii. tig. 9.

Diastopora Wrighti, Haime, 1854, op. cit. p. 186, pl. viii. fig. 5.

Diastopora foliacea (non Lamx.), Morris, 1843, Cat. Brit. Foss. p. 35 (fide Haime).

Diastopora Terquemi, Haime, 1854, op. cit. p. 187, pl. viii. figs. $7 a-d$.

Diastopora scobinula (non Mich.), Haime, op. cit. p. 186, pl. viii. fig. 6.

Mesenteripora scobinula (non Mich.), Sauvage, 1889, Bry. Jur. Boul., Bull. Soc. géol. France, sér. 3, t. xvii. p. 49.

Diagnosis.-Zoarium loose, composed of broad and fairly flat fronds; bilaminate.

Zoxcia visible throughout their length or almost so; their length is medium, and in shape they are regularly cylindrical.

Peristomes slightly raised, but not reflexed; they are mostly arranged in fairly regular oblique lines.

Formula. $-102 r 0$.

Distribution.-England: Inferior Oolite and Great Oolite. Foreign: Bajocian, France and Germany; Bathonian, France.

\section{Diastopora Michelini (Blainville), 1830.}

Mesenteripora Michelini, Blainville, 1830, Dict. Sci. nat. t. Ix. p. 397.

Diastopora Michelini, M.-Edwards, 1838, Mém. Cris., Ann. Sci. nat. Zool. sér. 2, t. ix. p. 226, pl. xiii. fig. 1 .

Bidiastopora Michelini, d'Ol'bigny, 1849, Prod. Pal. t. i. p. 317.

Mesenteripora dadalea, Blainville, 1830, Dict. Sci. Nat. t. lx. p. 397.

Diastopora foliacea (non Lamx.), Michelin, 1846, op. cit. p. 239, pl. Ivi. fig. 8.

Bidiastopora microphylla, d'Orbigny, 1849, Prod. Pal. t. i. p. 317.

Mesenteripora microphylla, d'Orbigny, 1852, Pal. franç., Terr. crét. t. v. p. 808 .

Diastopora microphylla?, Haime, 1854, Bry. Jur., Mém. Soc. géol. France, sér. 2, t. v. p. $19 \mathrm{i}$.

Bidiastopora latifolia, d’Orbigny, 1852, Pal. franç., Terr. crét. t. v. p. 799.

Diastopora latifolia, Haime, 1854, op. cit. p. 191.

? Diastopora conferta, Reuss, 1867, Bry. braun. Jura Balin, Denks. k. Akad. Wiss. Wien, Bd. exxvii. p. 10, pl. ii. fig. 6.

Diagnosis.-Zoarium hemispherical, formed of interlocking contorted bilaminate fronds. The surface appears cerebriform, as the sinuous edges of the fronds are separated by narrow depressions. end.

Zoccia short and very crowded, only visible at the distal 
Peristomes well raised; zocecia fusiform. The distribution of the peristomes is along irregular curved lines; but in some places the linear arrangement is not apparent.

Formula. $-211^{\prime \prime}(r) 2$.

Distribution.-England: Inferior Oolite-Forest Marble. Foreign : Bajocian, Germany ; Bathonian, France and Austria.

\section{Diastopora lamellosa, Mich., 1816.}

Diastopora lamellosa, Michelin, 1846, Icon. Zooph. p. 241, pl. lvi. tig. 11.

Mesenteripora lamellosa, Sauvage, 1889, Bry. Jur. Boul., Bull. Soc. géol. France, sêr. 3, t. xvii. p. 50.

Non Bidictstopora lamellosa, d'Orbigny, 1850, Prod. Pal. t. ii. p. 266.

Eschara Ranvilliana, Michelin, 1816, op. cit. p. ⒉43, pl. lvii. fig. 12.

Elea Ranvilliana, d'Órbigny, 1852, Pal. franç, 'Terr. crét. t. v. p. 628.

Lateromultelea Ranvilliana, d'Orbigny, 1852, op. cit. p. 629.

Jiastopora cervicornis, Michelin, 1846, op . cit. p. 241, pl. lvi. fig. 12.

Bidiastopora cervicornis, d'Orbigny, 1849, Prod. Pal. t. i. p. 317.

Elea cervicornis, d'Orbigny, 1852, Pal, fraņ̧, Terr. crét. t. v. p. 628.

Bidiastopora ramosissima, d'Orbigny, 1849, Prod. Pal. t. i. p. 317.

Diastopora ranosissima, Haime, 1854, op cit. p. 190, pl. ix. fig. 3 .

Elea ramosissima, d’Orbigny, 1852, Pal. franç., Terr. crét. t. v. p. 623.

Bidiastopora luciana, d’Orbigny, 1849, Prod. Pal. t. i. p. 317.

Multisparsa luciana, d'Orbigny, 1852, op. cit. p. 870, pl. 761. figs. 1-3.

Diastopora luciana?, Haime, 1854, op. cit. p. 191.

Diastopora fenestrata, Reuss, 1867, Bry. braun. Jura Balin, Denks. k. Akad. Wiss. Wien, Bd. cxxvii. p. 11, pl. ii. fig. 5.

Diagnosis. - Zoarium bilaminate, forming either thin, broad, crumpled fronds or thick narrow branches.

Zocecia short and broad, fusiform.

Peristomes slightly raised, not crowded, quincuncially arranged.

Formula.-1 21 r 0 .

Distribution.-England: Inferior Oolite-Bradford Clay. Foreign: Bajocian, Germany; Bathonian, France and Austria.

\section{Diastopora calloviensis (d'Orbigny), 1852.}

Elea calloviensis, d’Orbigny, 1852, Pal. franç., Terr. crét. t. v. p. 629.

Diastopora calloviensis, Haime, 1854, Bry. Jur., Mem. Soc. géol. France, sér. 2, t. v. p. 191.

? Diastopora subramosa, Wangen, 1868, Zone Amm. Sowerbyi, Geogn. Pal. Beitr. Bd. i. Heft 3, p. 645, pl. xxxiii. figs. 9, 10.

Diagnosis.-Zoarium of loose tufts, composed of bands which branch irregularly; bilaminate.

Zocecia long, broad, and regular in shape. 
Peristomes distant and well raised, irregularly distributed; zcoecia visible throughout their length.

Formula.-1" $02^{\prime \prime} i 0$.

Distrubution.-Fngland : Inferior Oolite, near Leckhampton. Foreign: Bathonian and Callovian, France.

\section{Diastopora Lamourouxi, M.-Edw.}

Diastopora Lamourouri, M.-Edwards, 1838, Mém. Crís., Ann. Sci. nat. Zool. sér. 2, t. ix. p. 225 , pl. xv. figr. 2.

Diastopora foliacea, pars, Lavtuuronx, 1821, Expos. móth. pl. 1xxiii. tig. 3.

Diastopora Waltoni, Haime, 185̃4, Bry. Jur., Mém. Soc. géol. France, sér. 2 , t. v. p. 184, pl. viii. fígs. $2 a, b$.

Diagnosis.-Zoarium unilaminate, growing in irregular tubes, which may branch repeatedly or broaden into funnelshaped expansions.

Zoocia usually visible throughout, the zoocia being long and the apertures usually distant.

Peristomes (in well-preserved specimens) highly raised and irregular in distribution, though occasionally there is a tendency towards a quincuncial system. Zocecia regularly cylindrical.

Formula.-3 02 i 0 .

Distribution.-England: Inferior Oolite. Foreign: Bajocian, Germany ; Bathonian, France and Austria.

\section{Synopsis of Species.}

I. Zocecia cylindrical.

A. Zoarium bilaminate.

a. Zocecia visible throughout.

Zoarium frondose:

peristomes well raised and their distribution irregular . .................... foliacea. peristomes slightly raised and their distribution subregular................... Davidsoni.

Zoarium ribbon-shaped:

peristomes raised and their distribution irregular $\ldots \ldots \ldots \ldots \ldots \ldots \ldots \ldots \ldots \ldots$ calloviensis.

b. Zooecia visible at ends $\ldots \ldots \ldots \ldots \ldots \ldots \ldots \ldots \ldots$ Michelini.

B. Zoarium unilaminate ................... Lamourouxi.

II. Zoocia lozenge-shaped ................ lamellosa. 